Case

Report

\title{
Simultaneous Stapling of Pulmonary Vein and Bronchus in Video-Assisted Thoracic Surgery Lobectomy
}

\author{
Guangliang Qiang, MD, ${ }^{1,2}$ and Jun Nakajima, MD, $\mathrm{PhD}^{2}$
}

\begin{abstract}
We present the case of a 66-year-old female who had undergone pulmonary wedge resection for diagnosis and followed by a radical lobectomy for the treatment of the primary lung cancer. We performed a left upper lobectomy through Video-Assisted Thoracic Surgery (VATS), we successfully adapted simultaneous stapling technique to division of both pulmonary vein and lobar bronchus which were densely adhered each other because of the previous surgery.
\end{abstract}

Keywords: simultaneous stapling, pulmonary vein, bronchus, VATS, lobectomy

\section{Introduction}

Video-Assisted Thoracic Surgery (VATS) has become common surgical procedure for treatment of lung cancer. Thoracic surgeons often encounter difficulty in lung cancer surgery when hilar vessels and bronchi are involved by direct tumor invasion or dense fibrous adhesion. We herein report a successful VATS lobectomy with the left upper pulmonary vein and upper lobe bronchus stapled at once for a patient who had previously undergone pulmonary resection.

\section{Case Report}

A 66-year-old woman was admitted to our hospital for surgical treatment of the lung cancer. She had undergone surgical therapy for breast cancer for 6 years. During follow up, in May 2012, chest computed tomography (CT) scan found a nodule of $3 \mathrm{~mm}$ in the left upper lobe.

${ }^{1}$ Department of Thoracic Surgery, China-Japan Friendship Hospital, Beijing, China

${ }^{2}$ Department of Thoracic Surgery, The University of Tokyo Hospital, Tokyo, Japan

Received: February 26, 2014; Accepted: March 24, 2014

Corresponding author: Jun Nakajima, MD, PhD. Department of Thoracic Surgery, The University of Tokyo Hospital, 7-3-1 Hongo, Bunkyo-ku, Tokyo 113-8655, Japan

Email: nakajima-tho@h.u-tokyo.ac.jp

(C)2015 The Editorial Committee of Annals of Thoracic and Cardiovascular Surgery. All rights reserved.
It became enlarged to $8 \mathrm{~mm}$ after 15 months observation (Fig. 1). We performed a VATS wedge resection of left upper lobe to determine the pathological diagnosis of the nodule. During the surgery, we found the nodule was located very near to the left upper pulmonary vein (Fig. 2). The pathological diagnosis was the primary lung adenocarcinoma, $5 \mathrm{~mm}$ in diameter on the basis of immunohistochemistry. We planned the left upper lobectomy with nodal dissection through thoracoscopy 35 days after the previous operation. During the VATS lobectomy, transections of the pulmonary arteries (A1-5) were performed successfully as usual. However, the upper pulmonary vein was densely adhered to the upper bronchus because of the previous surgery. We could not encircle the pulmonary vein even after opening the pericardium. After confirming that no hilar nodes were involved by the cancer, we encircled the pulmonary vein and the bronchus in bulk and stapled them at once with a green-cartridge staple. The lobectomy was thus successfully performed through thoracoscopy. The pathological stage was IA with no lymph node metastasis $(0 / 18)$. The chest tube was removed on postoperative day (POD) 2, and the patient was discharged from hospital on POD 5. She has been doing well for 3 months postoperatively.

\section{Discussion}

Lewis and colleagues first reported VATS lobectomy with bulk hilar division with a linear cutting stapler. ${ }^{1)}$ 

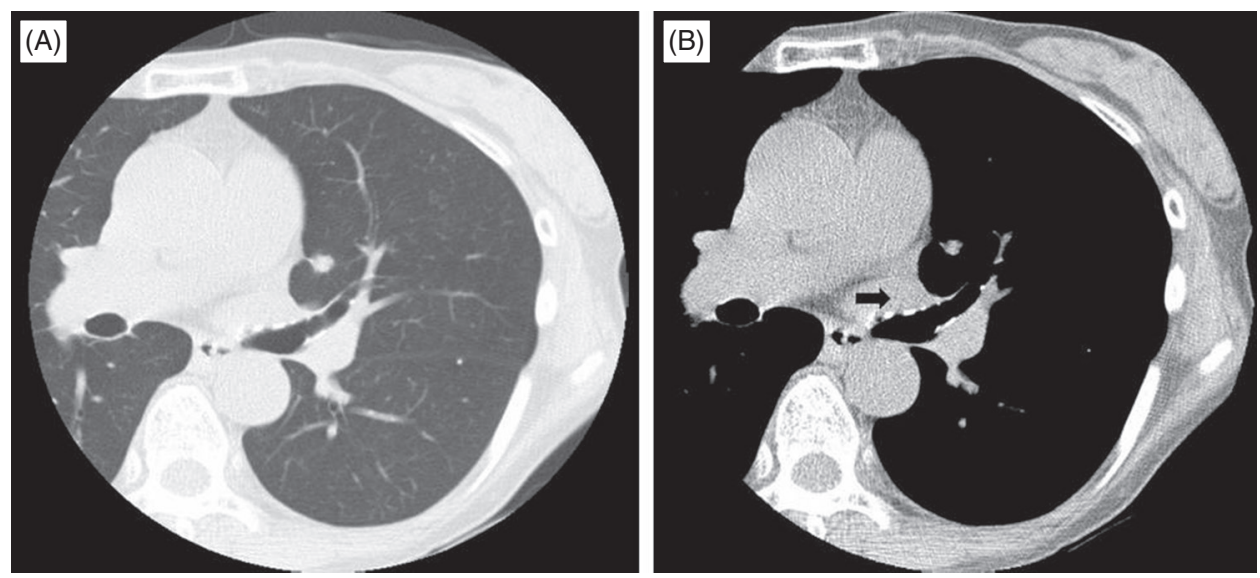

Fig. 1 Chest computed tomographic images taken before the wedge resection, showing (A) a nodule of $8 \mathrm{~mm}$ located in segment four of the left upper lobe, (B) the nodule was adjacent to the left superior pulmonary vein (black arrow).
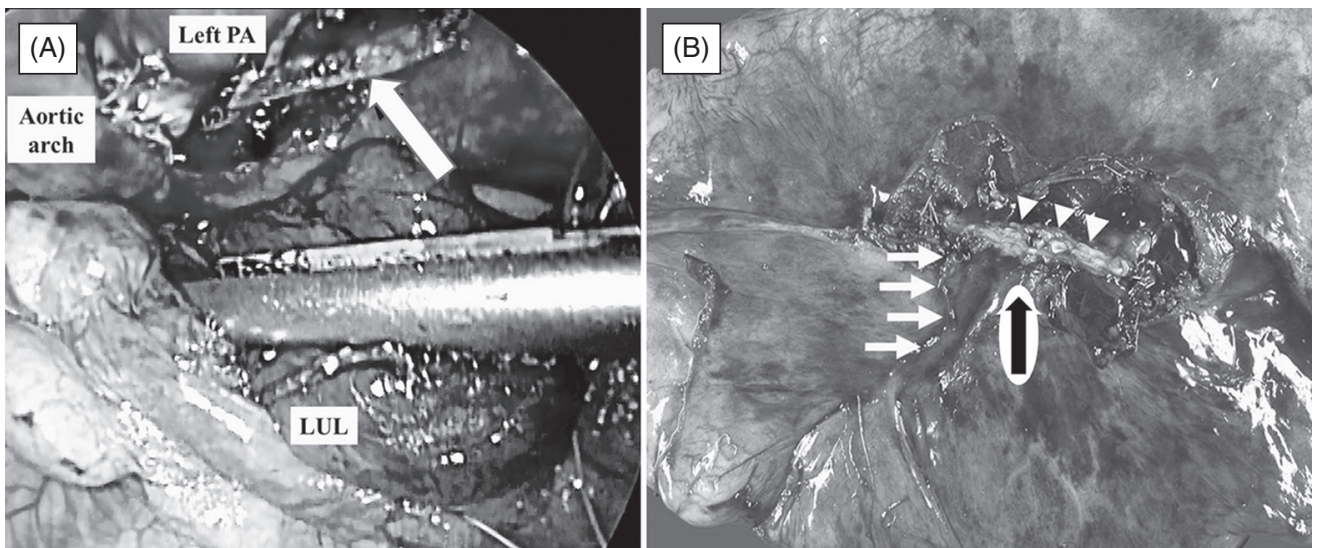

Fig. 2 Photographs of the simultaneous stapling of both pulmonary vein and lobar bronchus in left upper lobectomy. (A) the scene just after dividing both left upper bronchus and the left superior vein (white arrow). (B) from the medial view of the resected left upper lobe of the lung, previous stapling scar (white arrows) was very near to the left upper pulmonary vein (framed arrow) and bronchus (arrowheads). PA: pulmonary artery; LUL: left upper lobe.

This technique was also called "en masse" or "en bloc" lobectomy. Although excellent results using simultaneous stapling lobectomy or pneumonectomy have been reported, ${ }^{2-4)}$ it was not widely accepted to be a standard procedure because most of the surgeons feel that individual hilar management should the standard procedure in modern lobectomy. In spite of some concerns, this technique is still necessary.

Thoracic surgeons have disadvantages in dissection of hilar vessels or bronchi with dense adhesion through thoracoscopy. They might occasionally convert thoracoscopy to open thoracotomy to secure the safe lobectomy and avoid severe pulmonary vascular injuries. Simultaneous stapling technique could be an alternative method to overcome these difficulties. We had experienced simultaneous stapling of the pulmonary artery and bronchus in the past, as other author reported. ${ }^{4,5)}$ However, en masse stapling of pulmonary veins and bronchi has not been reported. In selected cases of VATS lobectomy, if a surgeon cannot separate the vascular or bronchial structures and staple them individually, an en masse lobectomy may be considered rather than conversion to an open thoracotomy to attempt more aggressive, more controlled approaches. But we must be aware of the shortage and potential complication of this technique and identify the inadequate cases for this approach. 
The first possible concern is the risk of hemorrhage. Adverse events of stapling are occasionally experienced in general thoracic surgery. Most severe intraoperative complication is vascular rupture and bleeding from the pulmonary stump during stapling. Actually there is no report of such complications while using this technique so far. With the development of autosuture device, the problem due to stapling failure is currently rare. Furthermore, the type of stapler should be chosen appropriately. We used a $4.8-\mathrm{mm}$ cartridge (green) considering the bronchus was involved, it is consistent with all of the other reports.

The second possible concern is the bronchopleural fistula (BPF) or bronchovascular fistula (BVF). BPF was underlined to lead to the maximum risk of mortality in patients with infectious disease. If infected bronchus was stapled with pulmonary vessels by this technique, the risk of BPF or BVF may be increased compared to the individual ligation technique. But this maybe only a logical surmise because there were no randomized controlled trials comparing between two methods. However, Lewis, et al. reported no bronchopleural or bronchovascular fistulas occurred in their practice of using this technique in 400 cases including infectious disease such as organizing pneumonias and aspergillus cavity with fungus ball. ${ }^{4)}$

The third possible concern is the validity for curative lung cancer surgery. Lewis, et al. evaluated the outcomes of the technique for 214 primary carcinomas, ${ }^{4)} 92$ patients were stage I, 104 patients were stage II, 9 patients were stage IIIA, and 9 patients were stage IV. The overall survival was $83 \%$ and none of the patients showed any recurrence at the stump site. The authors concluded that this technique conformed to the principles for curative resection of the lung cancer. However, according to the oncological basis, hilar lymph nodes should be completely dissected. But when pulmonary vessels and bronchus were simultaneously stapled the lymph nodes stuck in the stump could not be removed. So preoperatively evidence of negative lymph node involvement by means of mediastinoscopy or PET/CT is necessary to confirm that tight adherence is not due to metastasis. Every surgeon should consider mediastinal and hilar lymph nodes as potentially malignant and possibility of incomplete resection. In high risk cases surgical margins need to be confirmed, it's necessary to examine the frozen section of lymph nodes and/or the bronchial stump when simultaneously stapling is performed.

We herein present the simultaneously stapling technique in successfully dealing with the dense fibrous adhesion of upper pulmonary vein and bronchus. However, we suggest that the technique should only be used a last resort after isolating and severing as many hilar structures as possible. Furthermore, the knowledge of the potential risks may be helpful in decreasing adverse events.

\section{Disclosure Statement}

We do not have any financial support or relationships that may pose conflict of interest.

\section{References}

1) Lewis RJ. The role of video-assisted thoracic surgery for carcinoma of the lung: wedge resection to lobectomy by simultaneous individual stapling. Ann Thorac Surg 1993; 56: 762-8.

2) Amosov NM, Berezovsky KK. Pulmonary resection with mechanical suture. J Thorac Cardiovasc Surg 1961; 41: 325-35.

3) Lewis RJ, Caccavale RJ. Video-assisted thoracic surgical non-rib spreading simultaneously stapled lobectomy (VATS(n)SSL). Semin Thorac Cardiovasc Surg 1998; 10: 332-9.

4) Lewis RJ, Caccavale RJ, Bocage JP, et al. Videoassisted thoracic surgical non-rib spreading simultaneously stapled lobectomy: a more patient-friendly oncologic resection. Chest 1999; 116: 1119-24.

5) Kamiyoshihara M, Igai $H$, Ibe $T$, et al. Pulmonary lobar root clamping and stapling technique: return of the "en masse lobectomy". Gen Thorac Cardiovasc Surg 2013; 61: 280-91. 